\title{
Lagerungstechniken rund ums Knie - worauf ich in den Einzelfällen achten muss
}

Alexander Motzny

\section{Einleitung}

Die Rückenlagerung ist die meist gewählte Lagerung bei Frakturen/Operationen im Bereich des Kniegelenkes. Es haben sich jedoch aufgrund besserer Biomechanik neue OP-Techniken entwickelt, bei denen, vor allem bei Tibiakopffrakturen, auch eine Bauchlagerung notwendig wird. Auf diese beiden Techniken soll besonders eingegangen werden. Spezielle Lagerungen, z. B. für arthroskopische Eingriffe am Kniegelenk sowie Lagerungen zur Marknagelung im Bereich der proximalen Tibia und des distalen Femurs, sollen aber nicht unerwähnt bleiben.

\section{Grundsätzliches}

Der Patient sollte heute möglichst auf einem OPTisch mit Weichschaumauflage mit geteilten Beinplatten auf einer dünnen, wasserundurchlässigen Auflage gelagert werden. Zum scherkräftefreien Transfer des Patienten auf dem OP-Tisch verwenden wir zusätzlich eine sog. „Safetex-Unterlage“ (Inkontinenzunterlage) unter dem Schwerpunkt des Patienten. Das bedeutet: zwischen Schulter und Becken und nicht unter dem Becken ( $\bullet$ Abb. 1).

Es sollte für den Narkosearm eine Weichschaumarmschiene an der der Fraktur gegenüberliegenden Seite vorhanden sein. Ebenso sollte an dieser Seite der Narkosebügel befestigt werden. Für den Arm auf der Frakturseite ist eine geeignete Lagerung zu wählen. Wir verwenden dafür eine Klettarmfessel mit Polsterung, mit welcher der Arm auf dem Thorax, durch elastischen Bindenzug auf die Gegenseite, locker fixiert wird ( $\triangleright$ Abb. 2).

Auch ein Aufhängen am Narkosebügel ist denkbar, aber meist zu aufwendig. Eine 2. Armschiene auf der OP-Seite versuchen wir aus Platzgründen möglichst zu vermeiden. Bei der Armauslagerung sind Überstreckungen zur Schonung des Plexus brachialis zu vermeiden.

Zur Kopflagerung verwenden wir ein den Patientenbedürfnissen entsprechendes, geeignetes Kopfkissen und/ oder einen Weichschaumkopfring.

Der Patient sollte, so gut wie möglich, durch eine Röntgenschutzauf- bzw. -unterlage gegen Röntgenstrahlung geschützt sein, insbesondere im Bereich der Gonaden.
Um eine Auskühlung zu vermeiden, verwenden wir vorgewärmte Moltondecken zum Zudecken bereits beim Einschleusen des Patienten. Der Patient sollte während des Lagerungsvorganges so wenig wie möglich aufgedeckt werden. Bei längeren Eingriffen sollte ein geeignetes Wärmemanagement mit Wärmedecken und Wärmegeräten betrieben werden. Es wurde in verschiedenen Untersuchungen bewiesen, dass es einen direkten Zusammenhang zwischen der Auskühlung des Patienten und der Verträglichkeit der Narkose und der Infektionsrate gibt.

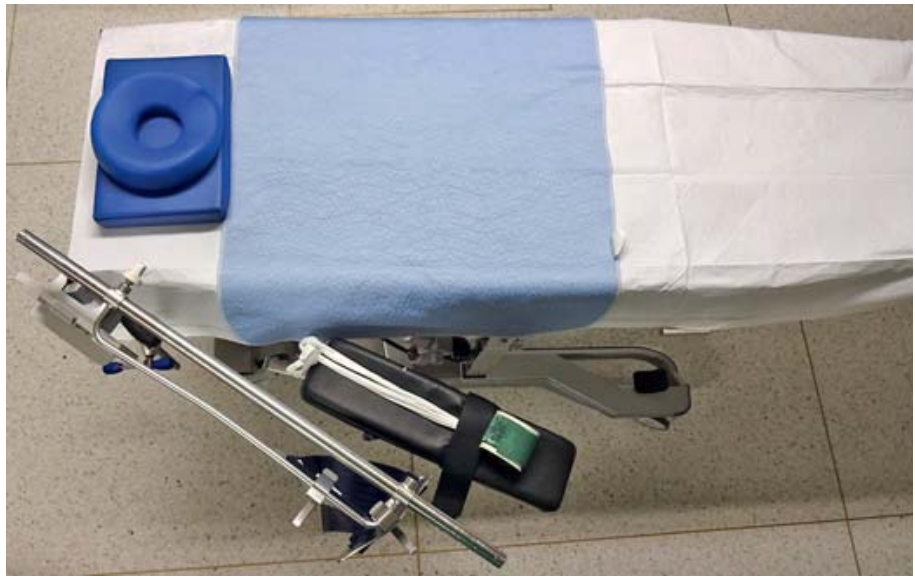

- Abb. 1 OP-Tisch mit Auflagen.

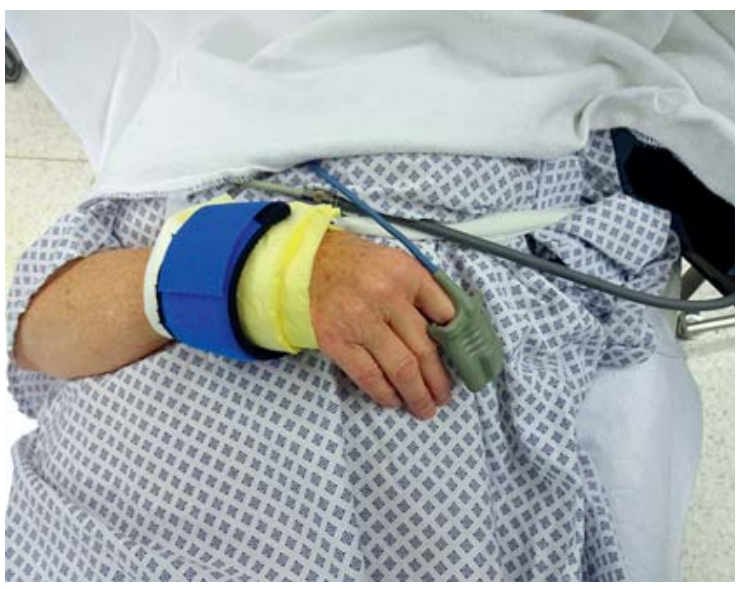

Abb. 2 Klettarmfessel. 


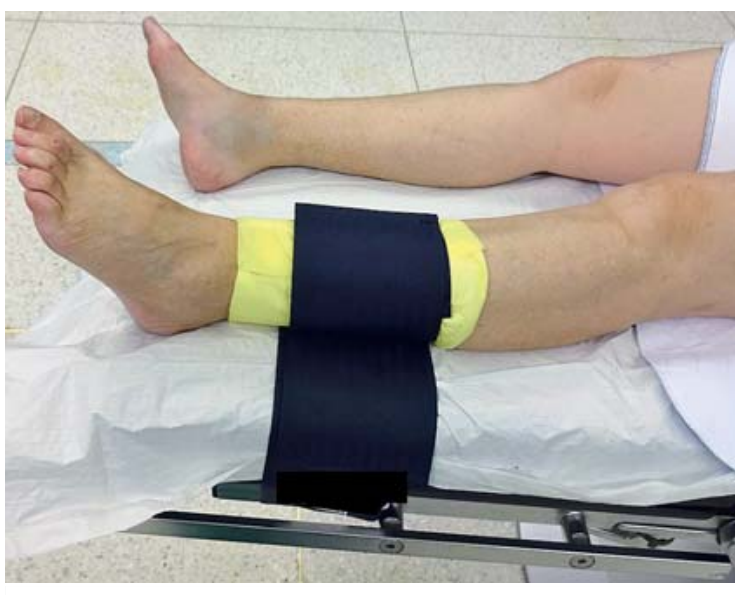

- Abb. 3 Beinfessel.

Eine Rasur des OP-Gebietes findet nicht mehr statt, das Kürzen der Haare mit einem Clipper reicht aus. Dies sollte idealerweise in der Narkoseeinleitung geschehen (Cave: Auskühlung). Der Patient wird grundsätzlich aus durchleuchtungstechnischen und hygienischen Gründen (weniger Metall im Strahlengang, Sterilabstand beim Abwaschund Abdeckvorgang) unter Verwendung der SafetexUnterlage vor Beginn des eigentlichen Lagerungsvorganges scherkräftefrei an das Fußende des OP-Tisches verbracht.

Das kontralaterale Bein wird mit einer gepolsterten Klettbeinfessel an der Beinplatte befestigt ( $\triangleright$ Abb. $\mathbf{3}$ ), um ein Herunterfallen des Beines intraoperativ zu vermeiden.

Eine Blutsperre, sofern benötigt, wird in geeigneter Weise mit Schutzfolie und Krepppapierbinde als Unterlage möglichst distal am Oberschenkel angelegt. Auf eine Polsterung mit Polsterwatte verzichten wir.

Eine Diathermieelektrode wird möglichst auf der Frakturseite an der Flanke oder am gegenüberliegenden Ober- schenkel gemäß Herstellerangaben aufgeklebt. Hierbei ist auf die Ableitung des Kabels zu achten, um Druckstellen zu vermeiden. Jeglicher Metall-Haut-Kontakt des Patienten mit dem OP-Tisch, Infusionsständer etc. ist bei Anwendung von monopolarem Strom zur Koagulation auszuschließen. Das nicht zu operierende Bein wird mit einer angewärmten Moltondecke zugedeckt. Ein wasserabweisendes Tuch wird unter das zu operierende Bein gelegt. Unter die Pobacke wird eine zu der nicht saugenden Seite gefaltete Windel als Saug- und Nässeschutz geschoben ( $\bullet$ Abb. 4 a, b).

\section{Rückenlagerung}

Die meist angewandte Lagerung bei Frakturen im Kniebereich ist die Rückenlagerung. Es wird ein Normaltisch oder Normaltisch mit Carbonbeinplatten (Tibia, Patella) bzw. ein Normaltisch mit Beinverlängerung (Femur) in Abhängigkeit vom Verfahren und von der Körpergröße des Patienten verwendet.

Bei der Versorgung mit Platten und Schrauben sowie bei einer Nagelung ist die seitliche Durchleuchtung von größter Bedeutung.

Hierfür existieren verschiedene Möglichkeiten der Optimierung:

- Ablassen der gegenüberliegenden Beinplatte und/ oder

- Unterpolsterung des Femurs unsteril unter der Abdeckung,

- Unterpolsterung des Femurs mit sterilen Lagerungshilfsmitteln.

- Diese sollten durchleuchtbar sein. Hier kommen verschiedene industrielle oder selbst entwickelte „Tools“ zur Anwendung (ohne Anspruch auf Vollzähligkeit!):

- zusammengefalteter OP-Mantel,

- Backtable Cover mit Klebestreifen fixiert = „Tübinger Bobbel“ ( $\triangleright$ Abb. 5),
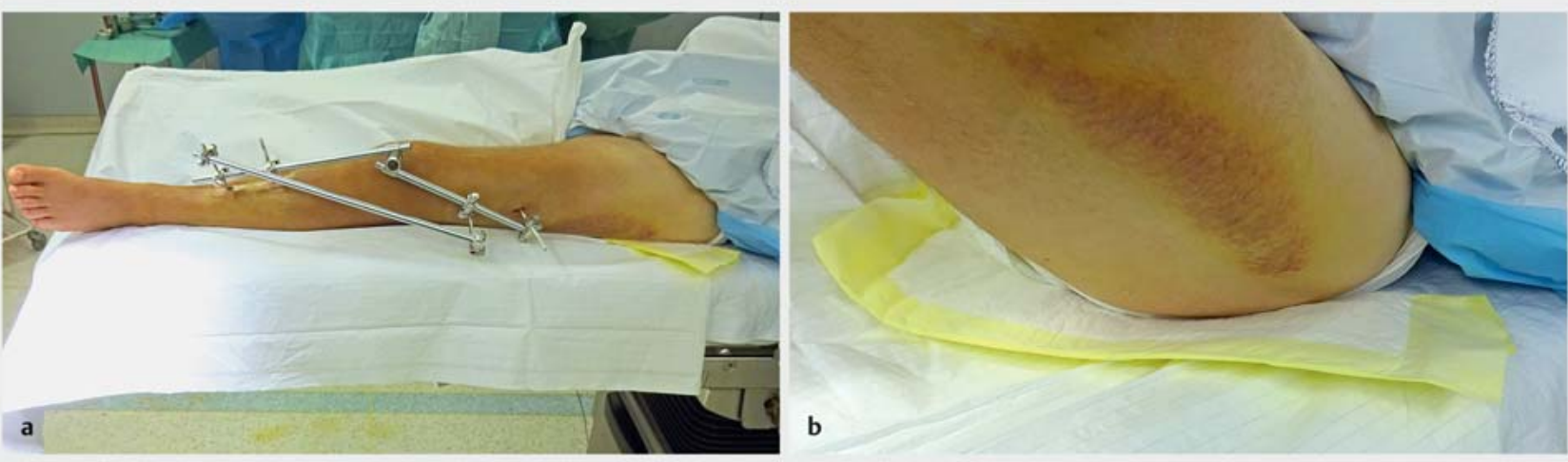

- Abb. 4 a wasserundurchlässige Unterlage. b saugfähige Windel. 
- sterilisierbare Kniebänke/Dreiecke in verschiedenen Größen aus Aluminium ( $>$ Abb. 6),

- Weichschaum mit Kunststoffummantelung etc.

- Unterpolsterung der Tibia mit einem Lagerungskissen

$\rightarrow$ Erhöhung der Beinplatte

Besonders zu beachten ist, dass diese Hilfsmittel nicht direkt in der Kniekehle zu liegen kommen, da es sonst zur Schädigung der dort verlaufenden Gefäße und Nerven durch Kompression kommen kann. Ein weiterer dadurch entstehender positiver Effekt, bedingt durch die leichte Beugung im Kniegelenk, ist die Entlastung des Muskelund Bandapparates des Oberschenkels und dadurch Aus-/Aufrichtung der Fraktur durch Ligamentotaxis.

\section{Sonderformen}

\section{Versorgung einer proximalen Tibiafraktur mittels Nagel}

- normale Rückenlage

- mit hängendem Bein, hierfür kann, muss aber nicht zwingend, die Beinplatte entfernt werden

- große/lange Kniebank zum Aufstellen des Beines

- L-/V-förmiges Gestell aus Fixateur-externe-Stangen und -Backen

- Cave: Gefäße und Nerven

- zusätzliche Polsterung notwendig

- L-Bügel steril oder unsteril als Gegendruckstab unter die Ferse oder steril zum An- und Abbauen.

- Auch eine Lagerung im Arthroskopiebeinhalter ist denkbar.

- Extensionstischlagerung, nur noch wenig in Gebrauch, sehr aufwendig, aber in speziellen Situationen sehr hilfreich. Als Standardverfahren nur geeignet bei häufiger und standardisierter Anwendung.

\section{Tipps und Tricks}

- Um Achse, Länge und Rotation optimal beurteilen zu können, decken wir gelegentlich beide Beine ab ( $\triangleright$ Abb. 7).

- Auch die Anlage und Ausrichtung mit einem Distraktor oder einem speziellen Distraktionsrahmen mittels Fixateur externe ist denkbar. Diese Methode ist bei instabilen Frakturen sehr hilfreich.

- Ebenso ist eine Nagelung bei liegendem Fixateur externe unter sukzessiver Entfernung desselben möglich.

\section{Versorgung einer distalen Femurfraktur mittels Nagel}

- normale Rückenlage möglich

- Am hängenden Bein, hierzu empfiehlt es sich, die Beinplatte zu entfernen und durch eine kürzere zu ersetzen, um eine optimale Auflage für das Femur zu erreichen.

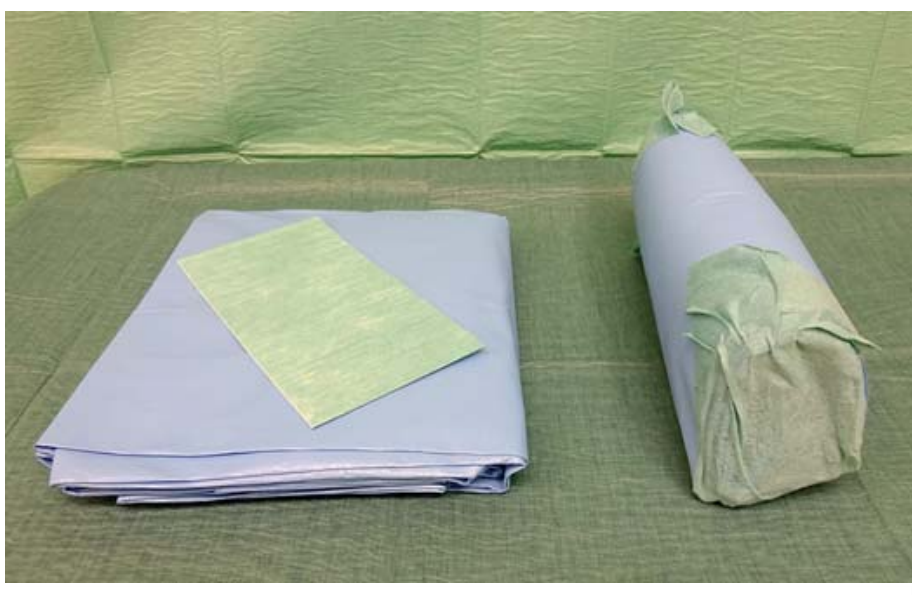

- Abb. 5 „Tübinger Bobbel“.

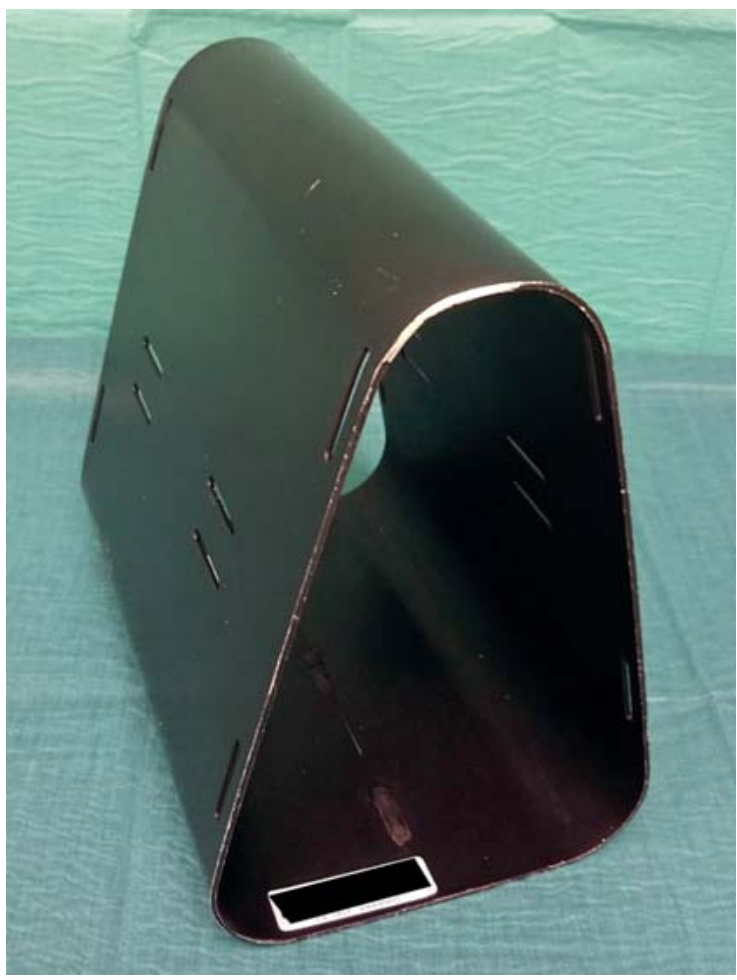

Abb. 6 Aluminiumkniebank, sterilisierbar.

\section{Tipps und Tricks}

- Beide Beine abdecken, um durch Anheben des gesunden Beines ein seitlich optimales Durchleuchten zu ermöglichen.

- Die Auslagerung des nicht betroffenen Beines in einer Göbel-Beinschale ist denkbar, um eine gute seitliche Durchleuchtung zu gewährleisten. Cave: N. peroneus am Fibulaköpfchen. Die Abdeckung wird bei dieser Lagerung etwas aufwendiger. 


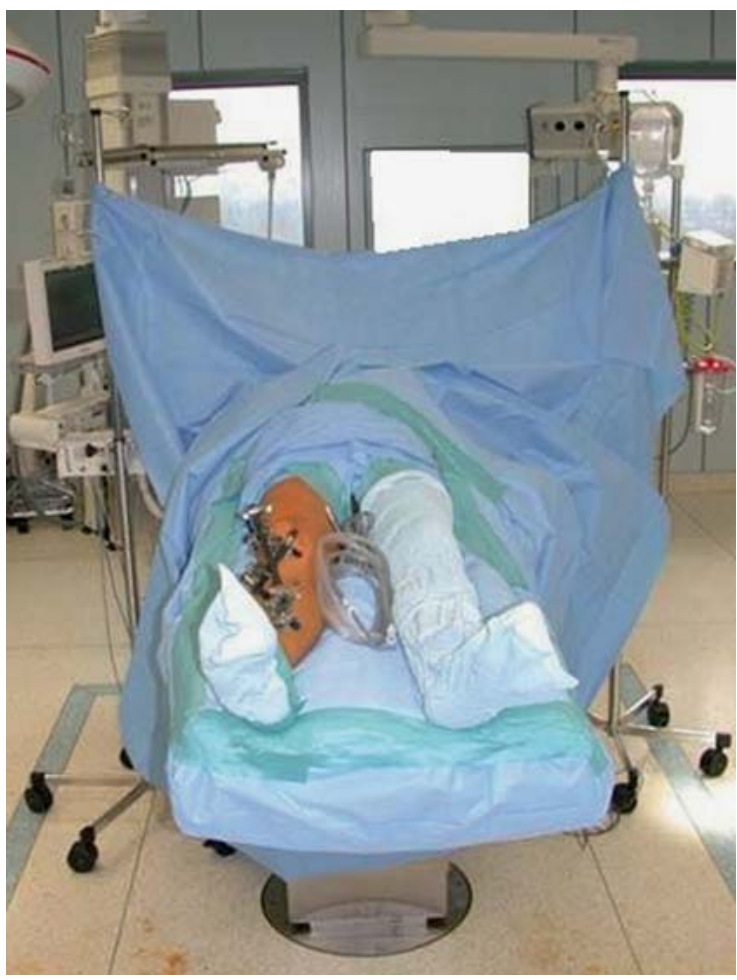

Abb. 7 Beidbeinige Abdeckung.

\section{Bauchlagerung}

Hier verwenden wir einen Normaltisch mit Weichschaumauflage und 2 Armschienen.

Wir drehen unseren Patienten grundsätzlich auf dem OPTisch in Bauchlage. Hierfür verwenden wir eine 2. SafetexUnterlage. Wir führen die Drehung des Patienten nach kinästhetischen Grundsätzen durch, sodass weder Kraft noch eine erhöhte Anzahl von Menschen hierfür notwendig ist!

In Bauchlage lagern wir genauso wie in Rückenlage (wasserundurchlässige Unterlage, Windel als zusätzlicher Nässesaugschutz etc.). Zur Fixierung des gesunden Beines kann eventuell ein 2. Beckenbeingurt notwendig werden.

\footnotetext{
Merke

Das Anlagern der Blutsperre empfiehlt sich vor dem Umdrehen des Patienten, da dies in Bauchlage sehr schwierig ist!
}

Sowohl der Anästhesiearm als auch der andere Arm werden auf einer Armschiene mit Weichschaumauflage gelagert. Hier ist besonders auf die Ober- und Unterarmausrichtung (nicht über $90^{\circ}$ ) zu achten. Ebenso sollte das Schultergelenk frei beweglich gelagert sein und kein Zug auf den Plexus brachialis ausgeübt werden.

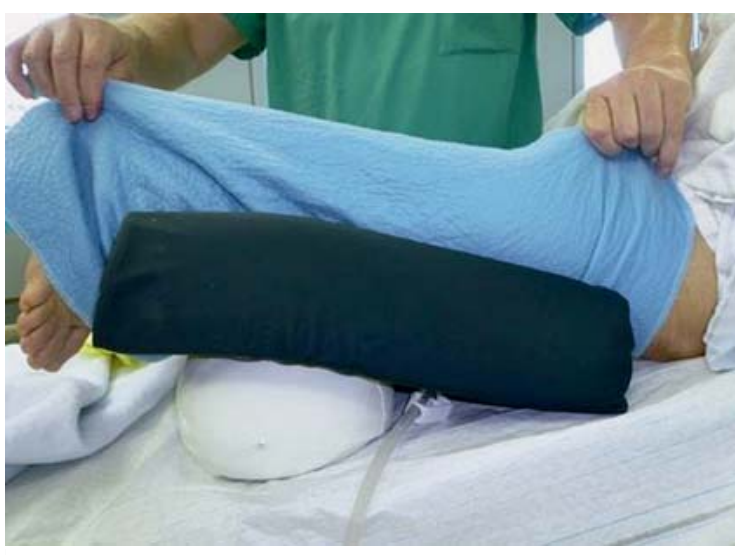

Abb. 8 Weichschaum-C-Schiene.

Das gegenüberliegende Bein kann ebenso, zur besseren seitlichen Durchleuchtung, mit der beweglichen Beinplatte abgelassen werden.

Besonders wichtig ist die Unterpolsterung des Fußes zur Freilagerung der Zehen zur Vermeidung von Druckschädigung. Besonders eignen sich hierfür konzipierte C-förmige Weichschaumschienen kombiniert mit einer Knierolle ( $\triangleright$ Abb. 8 ).

Die Unterpolsterung des Thoraxes und des Beckens führen wir in enger Absprache mit den Anästhesisten und eher puristisch durch. Hierzu heben wir den Patienten mit der OP-Tisch-Auflage an und schieben zusammengerollte Moltons unter den Patienten. Auch das Anheben mit dem Safetex und direkte Unterpolsterung ist möglich. In diesem Fall verwenden wir besondere Flockenkissen ( $\triangleright$ Abb. 9).

Merke

Falten und Kabel, Urinkatheter unter dem Patienten. Ebenso sollte bei Männern auf die Lage des Penis geachtet werden, um hier Druckschäden zu vermeiden.

Alternativ sind jegliche Art von Bauchrahmen denkbar. Diese Art der Thorax- und Beckenlagerung ist jedoch nur mit einem 2. OP-Tisch und dem Drehen von diesem auf den vorgerichteten OP-Tisch im Saal rückenschonend möglich. Logistisch sehr aufwendig und material- und personalintensiv bei höherem Kraftaufwand im Gegensatz zu unserer „Safetex-Methode“.

Für die Kopflagerung empfiehlt sich ein Weichschaumkopfring oder eventuell gar nichts, direkte Lagerung des Kopfes, zur Seite gedreht, auf der Weichschaum-OPTisch-Auflage und ggf. etwas Ablassen der OP-TischKopfplatte zur Entlastung der Halswirbelsäule. 


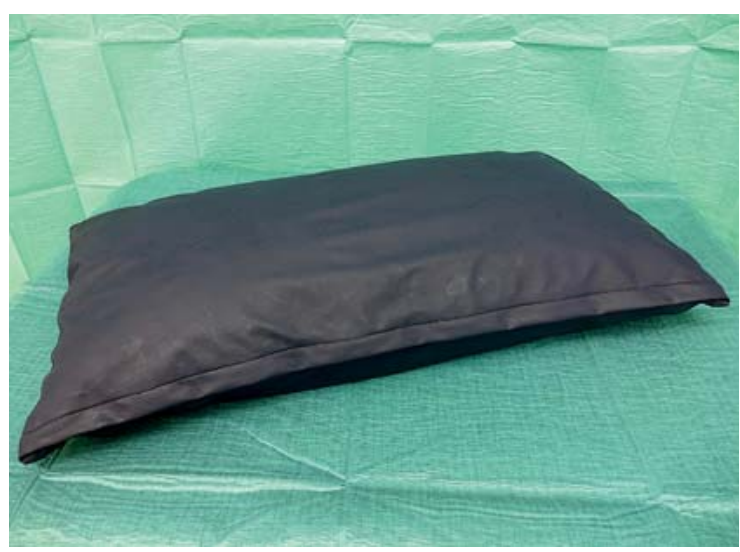

- Abb. 9 Flockenkissen.

Kopf- und Armlagerung führen wir immer in enger Absprache mit dem Anästhesieteam und unter Berücksichtigung der individuellen Patientenbedürfnisse durch.

\section{Lagerungen am Kniegelenk für arthroskopische Eingriffe}

Es wird ein Normaltisch mit Weichschaumauflage verwendet, siehe Rückenlage.

Wir verwenden bei arthroskopischen Eingriffen am Kniegelenk einen starren Beinhalter, der am OP-Tisch befestigt wird. Ganz besonders wichtig sind in diesem Fall die Gonaden, da es aufgrund der Ausladung des Beinhalters zu Druckproblemen in diesem Bereich kommen kann. Der Metallkontakt ist aus Erdungsaspekten immer auszuschließen. Die Beinplatte wird entfernt. Das gesunde Bein wird, wie üblich, mit Beinfessel gelagert und die Beinplatte wird $10-15^{\circ}$ abduziert. Einerseits, um Platz zu gewinnen, und andererseits, um Entlastung am Beinhalter zu bekommen ( $\triangleright$ Abb. 10).

Ein Verstellen der Beinlagerung ist dadurch nach der Abdeckung sehr schwierig. Bei idealer Befestigung am OPTisch ist ein Auf- und Abbewegen mit der OP-Tisch-Fernbedienung möglich.

Manchmal ist es notwendig (besondere Zugangswege, Durchleuchtung), das gesunde Bein in einer Göpel-Schale auszulagern. Dabei werden beide Beinplatten entfernt. Es besteht auch die Möglichkeit der Beinlagerung in einem speziellen Beinlagerungsaggregat, welches intraoperativ das Aufstellen und Absenken des Beines via Fußschalter ermöglicht ( $\triangleright$ Abb. 11).

Für kurze arthroskopische Eingriffe am Knie verwenden wir lediglich eine Gegenstütze auf Höhe der Blutsperre am Oberschenkel. Die Beinplatte wird nicht entfernt, sondern nur etwas abgelassen ( $\bullet$ Abb. 12).



- Abb. 10 Arthroskopiebeinhalter hängendes Bein.

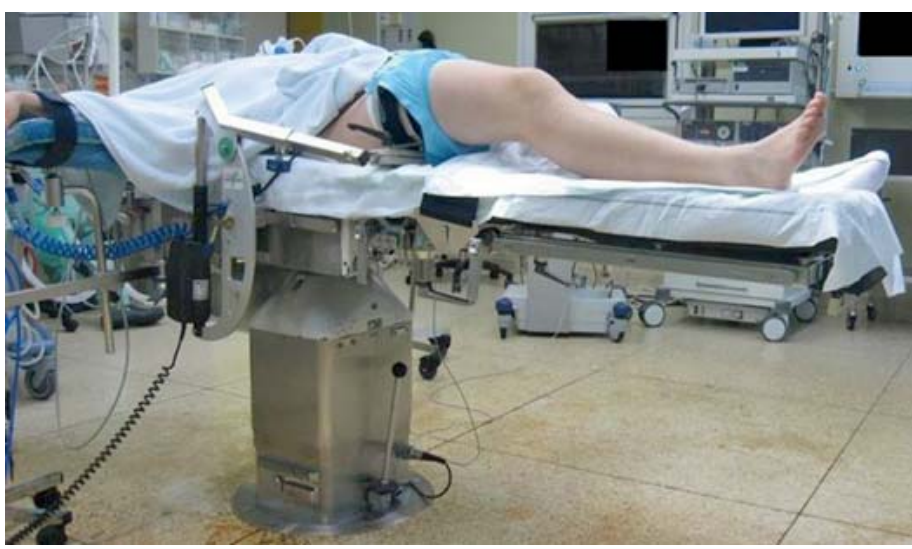

- Abb. 11 Hydraulisches Beinlagerungsaggregat.

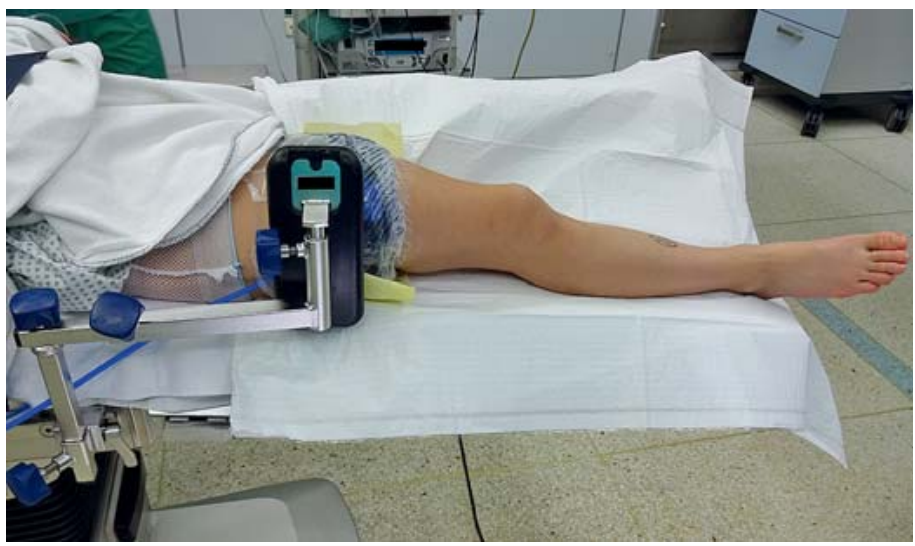

- Abb. 12 Gegenstütze als Hypomochlion.

\section{Zusammenfassung}

Die große Kunst der Lagerung, nicht nur am Kniegelenk, beruht grundsätzlich auf Erfahrung und klaren Lagerungsstandards. In Sondersituationen und bei besonderen Indikationen bedarf es der klaren Absprache zwischen 
Operateur, Anästhesist und OP-Personal, um gemeinsam, mit viel Erfahrung und Gefühl für die Bedürfnisse des Patienten und zur Schaffung optimaler OP-Bedingungen, das beste Lagerungsergebnis zu erzielen.

Interessenkonflikt

Die Autoren geben an, dass kein Interessenkonflikt besteht.

Autor

\section{Alexander Motzny}

Stv. OP-Leitung/Leitung AEMP, Mentor AOTrauma ORP Deutschland, BG Unfallklinik Tübingen
Korrespondenzadresse

\section{Alexander Motzny}

BG Unfallklinik Tübingen

Schnarrenbergstraße 95

72076 Tübingen

Tel.: 0 7071/606-3090

Fax: 07071/606-1070

amotzny@bgu-tuebingen.de

Bibliografie

DOI https://doi.org/10.1055/a-0819-2863

OP-JOURNAL 2019; 35: 181-186 @ Georg Thieme Verlag KG Stuttgart · New York ISSN 0178-1715 